\title{
Experimental and numerical study on surface roughness of magnetorheological elastomer for controllable friction
}

\author{
Rui LI ${ }^{1,2}$, Xi LI ${ }^{1,2}$, Yuanyuan $\mathbf{L I}^{2}$, Ping-an YANG ${ }^{1,2, *}$, Jiushan LIU ${ }^{1,2}$ \\ ${ }^{1}$ Key Laboratory of Industrial Internet of Things \& Networked Control, Ministry of Education, Chongqing University of Posts and \\ Telecommunications, Chongqing 400065, China \\ ${ }^{2}$ School of Automation, Chongqing University of Posts and Telecommunications, Chongqing 400065, China \\ Received: 02 February 2019/Revised: 03 April 2019 / Accepted: 03 June 2019 \\ (C) The author(s) 2019.
}

\begin{abstract}
Magnetorheological elastomer (MRE) is a type of smart material of which mechanical and electrical properties can be reversibly controlled by the magnetic field. In this study, the influence of the magnetic field on the surface roughness of MRE was studied by the microscopic modeling method, and the influence of controllable characteristics of the MRE surface on its friction properties was analyzed by the macroscopic experimental method. First, on the basis of existing studies, an improved mesoscopic model based on magnetomechanical coupling analysis was proposed. The initial surface morphology of MRE was characterized by the $\mathrm{W}-\mathrm{M}$ fractal function, and the change process of the surface microstructures of MRE, induced by the magnetic interaction between particles, was studied. Then, after analyzing the simulation results, it is found that with the increase in the magnetic field and decrease in the modulus of rubber matrix, the surface of MRE changes more significantly, and the best particle volume fraction is within $7.5 \%-9 \%$. Furthermore, through experimental observation, it is found that the height of the convex peak on the surface of MRE decreases significantly with the action of the magnetic field, resulting in a reduction in the surface roughness. Consistent with the simulation results, a particle volume fraction of $10 \%$ corresponds to a maximum change of $14 \%$. Finally, the macroscopic friction experiment results show that the friction coefficients of MREs with different particle volume fractions all decrease with the decrease in surface roughness under the magnetic field. When the particle volume fraction is $10 \%$, the friction coefficient can decrease by $24.7 \%$ under a magnetic field of $400 \mathrm{mT}$, which is consistent with the trend of surface roughness changes. This shows that the change in surface morphology with the effect of the magnetic field is an important factor in the control of MRE friction properties by magnetic field.
\end{abstract}

Keywords: controllable friction; surface roughness; magnetorheological elastomer (MRE); mesoscopic model; coupled magneto-mechanical analysis; numerical simulation

\section{Introduction}

Magnetorheological elastomer (MRE) [1, 2] is a new type of smart material composed of polymer matrix mixed with micron-sized magnetic particles. The mechanical and electrical properties of MRE can be controlled by an external magnetic field. Because of these adjustable functions, MRE has exhibited advantages over traditional rubber materials in active/semi- active vibration control, such as in bridges, automotive vehicles, and engine mounts [3-6]. Thus far, the controllable stiffness and damping characteristics of the MRE have been applied in the field of vibration isolation. As a versatile smart engineering material, researchers have attempted to apply MRE to new fields and found that MRE exhibits excellent magnetic control properties as well as potential for applications in the fields of noise reduction, smart sensing,

* Corresponding author: Ping-an YANG, E-mail: yangpa@cqupt.edu.cn 
electromagnetic shielding, and friction regulation [7-11]. In particular, the magnetic control friction characteristics of MRE provide a new way to actively control the friction characteristics of materials or devices, promoting the development of smart instruments and equipment.

MRE is a mixture of metal and viscoelastic matrix. On the one hand, it provides the possibility for magnetic field to control friction and reduce wear; on the other hand, because of the influence of the magnetic field, its friction mechanism becomes more complex. Factors such as hardness, elastic modulus, surface topography, chemistry, vibration, and temperature all contribute to the complex contact conditions, leading to a change in the tribological characteristics [12-15]. To explore the magnetically controlled friction characteristics of the MRE under the action of a magnetic field, Lee et al. [11] independently designed and built a sliding friction test platform. The friction characteristics of MRE, which is filled with $\mathrm{Fe}, \mathrm{Ni}$, and Co powders, were tested under magnetic and non-magnetic conditions. The results showed that the friction coefficient of MRE would change significantly because of the magnetic field. At the same time, the microstructure analysis showed that magnetic particles would accumulate on the surface under the magnetic field. They argue that the aggregation of these particles increases the resistance of MRE, changing the friction and wear properties. Furthermore, the variable friction characteristics of MRE were described by the same group [16]. They found that the hardness of all MRE samples increased to varying degrees after application of the magnetic field, resulting in a change in the friction and wear characteristics of MRE. In addition, Lian et al. $[17,18]$ have also carried out experiments to study the effects of some external environmental factors on the friction characteristics of MRE. The research shows that vibration, temperature, and relative humidity all affect the friction characteristics of MRE. Previous studies have mainly observed and explained the adjustable friction performance of MRE from the perspective of macroscopic experiments, and conclude that the frictional coefficient change of MRE under a magnetic field was due to the field-stiffening effect. However, the manner in which the magnetic field influences the friction coefficient of MREs with different particle volume fractions has not been studied. All experiments are carried out under conditions of magnetic field and no magnetic field, in which the effect of different magnetic fields on the friction performance of MRE is not reflected. In terms of mechanism exploration, the change in the MRE friction coefficient is explained only from the perspective of mechanical property change. The consideration of other influencing factors is lacking, and the mechanism is still unclear.

Recently, some researchers [19-22] have found that the surface microstructures of MRE will change under the influence of the magnetic field, which provides a new idea for exploring the mechanism of magnetroncontrolled friction of MRE. In previous studies [23], we found that the surface roughness of MRE would change under the action of the magnetic field. However, the observation of MRE surface topography is only from a two-dimensional perspective; thus, the discussion about which factors affect the change trend of roughness is not comprehensive. The effect of the change in surface roughness caused by the magnetic field on the friction coefficient is unknown.

Therefore, in this study, the changes in surface morphology and the friction coefficient of MRE under the external magnetic field are studied using the methods of microscopic modeling and macroscopic experiment, and the relationship between the surface morphology and friction is expounded. The effects of internal factors (particle volume fraction, matrix modulus) and external excitation (magnetic field intensity) on the surface morphology of materials are discussed. The MRE samples with different volume fractions are fabricated, and their surface morphology and friction properties are tested under different magnetic fields. Finally, according to the theoretical analysis and experimental results, the effect of surface morphology on the friction characteristics of MRE is briefly described.

\section{Numerical simulation}

Under dry friction conditions, the interface morphology of materials is one of the main factors affecting friction performance. Although most of the studies have observed the surface microstructures of MRE by experimental methods, it is difficult to observe the 
reasons for the change in surface microstructures and of which parameters would possibly influence the change in the surface through just experiments. It is known that the rubber matrix of MRE is not affected by the magnetic field. The change in the performance is mainly attributed by adding magnetic particles. Therefore, in the following section, we discuss a theoretical simulation from a microscopic perspective to investigate the magnetic field inducing the interaction of ferromagnetic particles inside MRE, and consequently, the change in surface microstructures of MRE.

\subsection{Development of the finite element (FE) model}

Based on the actual surface microstructure of the MRE samples, we proposed a numerical model for isotropic MRE and assumed that all the magnetic particles have the same radius and are tightly bonded with the matrix. Because the magnetic induced deformation of MRE is usually very slight [24, 25], the matrix is considered as an incompressible linear elastic material. The FE model of the isotropic MRE material with randomly distributed particles in two dimensions is shown in Fig. 1, where the round points represent the magnetic particles, and the grey portion is the rubber matrix.

The number of particles is set to 200, which is equivalent to a volume fraction of about $10 \%$, and the diameter of the particles is chosen to be $3 \mu \mathrm{m}$. By varying the particle number, different particle volume

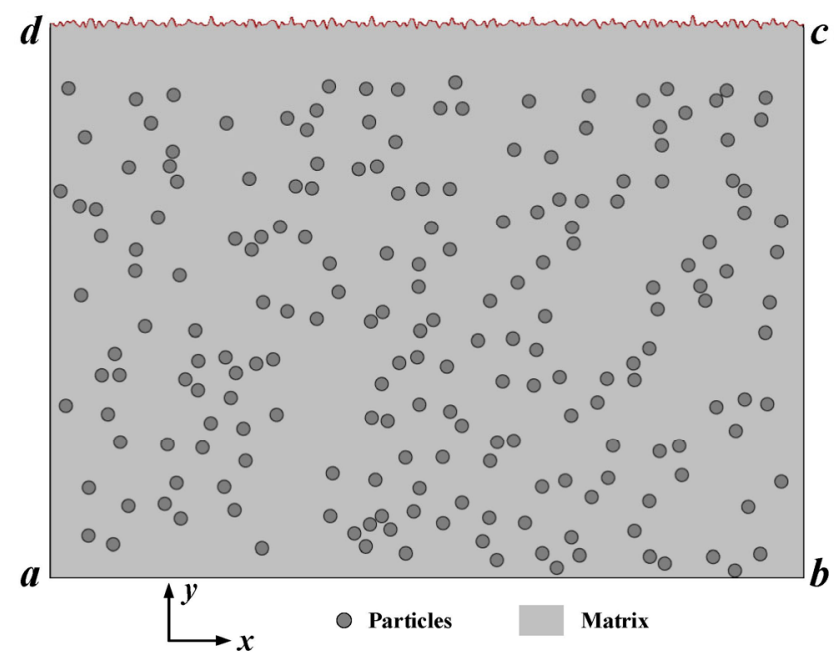

Fig. 1 The two-dimensional FE model of MRE. fractions can be achieved. Because of the preparation with an unsmoothed mold, the surface of MRE exhibits a certain roughness, which can be confirmed by our previous study [23]. Therefore, the initial surface microstructure of MRE should be considered in the study of surface morphology changes under the magnetic field. The rough surface topography of the material is commonly described by fractal geometry, as this is characterized by the properties of continuity, non-differentiability, scale invariance, and self-affinity [26]. In the model, the surface profile of MRE is described by fractal geometry, and its two-dimensional surface profile height is given by the $\mathrm{W}-\mathrm{M}$ function [27]:

$$
y(x)=G^{D-1} \sum_{n=n_{1}}^{n_{2}} \frac{\cos 2 \pi \gamma^{n} x}{\gamma^{(2-D) n}}, 1<D<2
$$

where $D$ is the fractal dimension, which determines the complexity of the surface profile, $G$ is the fractalscale coefficient, which reflects the amplitudes of the surface profile, $\gamma$ is a constant $(\gamma=1.5$ is typical for most surfaces), and $n$ is the fractal-scale index.

In this work, parameter $R a$ is used to represent the value of the surface roughness. It can be expressed as follows:

$$
R a=\frac{1}{l_{c d}} \int_{0}^{l_{c d}}|y(x)| \mathrm{d} x=\frac{1}{n} \sum_{i=1}^{n}\left|y_{i}\right|
$$

where $y(x)$ is the contour height of each point on the surface profile of MRE, the measuring length is $l_{c d}, n$ represents the number of measurement points selected on the surface profile of length $l_{c d}$, and $y_{i}$ is the contour height of each measurement point. The roughness of MRE surface microstructures is determined by parameters $D$ and $G$.

Previous experimental studies [23] have shown that the initial surface roughness of MRE is $2-3 \mu \mathrm{m}$. The width of the rough surfaces to be modeled is limited to $150 \mu \mathrm{m}$, based on Eqs. (1) and (2), and the initial surface microstructure of the MRE is generated using 300 sampling points, as shown in Fig. 2. The expectation value of the fractal dimension $(D)$ is 1.58 , and the fractal-scale coefficient $(G)$ is 5.95 , while the initial surface roughness is calculated to be $2.66 \mu \mathrm{m}$. 


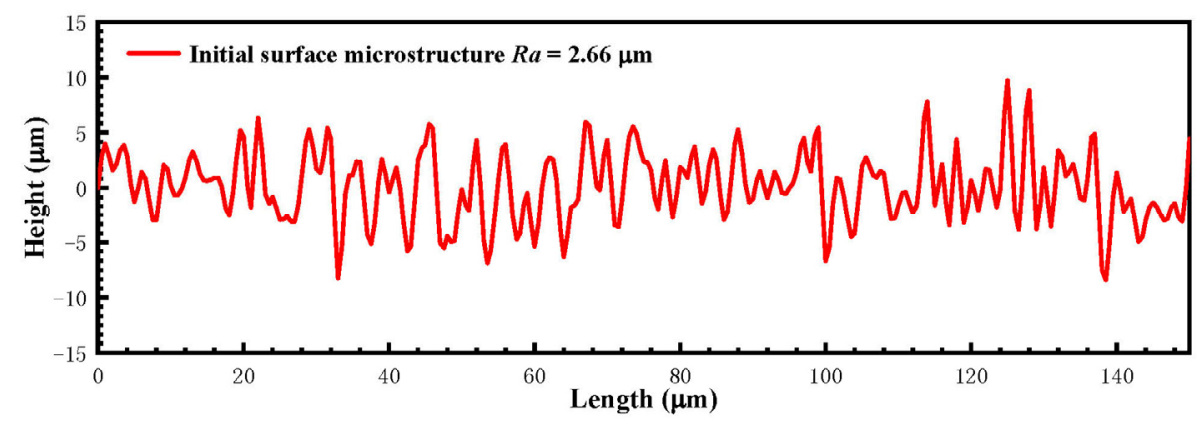

Fig. 2 The initial surface micro-profile of MRE.

\subsection{Coupled magneto-mechanical analysis}

In this study, the coupled magneto-mechanical/ displacement analysis was carried out to obtain the distribution of magnetic field and stress/strain inside MREs. Because constructing the direct coupling matrix is difficult, we use the sequential method to solve the magneto-mechanical coupling problem by using commercial software COMSOL Multiphysics. The coupled equations illustrated in matrix representation are [28]:

$$
\left[\begin{array}{cc}
{\left[K_{\mathrm{M}}\right]} & {[0]} \\
{[0]} & {\left[K_{\mathrm{S}}\right]}
\end{array}\right]\left\{\begin{array}{l}
{\left[D_{1}\right]} \\
{\left[D_{2}\right]}
\end{array}\right\}=\left\{\begin{array}{l}
{\left[f_{1}\right]} \\
{\left[f_{2}\right]}
\end{array}\right\}
$$

where $\left[K_{\mathrm{M}}\right]$ and $\left[K_{\mathrm{S}}\right]$ are the element matrices of the magnetic field and stress field respectively, $\left[D_{1}\right]$ and $\left[D_{2}\right]$ are displacement matrices, and $\left[f_{1}\right]$ and $\left[f_{2}\right]$ are excitation matrices from the two fields.

In the magnetic module, applying a uniform magnetic field in the $y$-direction of the model shown in Fig. 1, and assuming that the magnetic scalar potential of the bottom surface $l_{a b}$ is zero, the magnetic field intensity acting on the particles along the $y$-direction is $\mathbf{H}$, and the rest of the edges are magnetically insulated. After magnetization, an interaction force is generated between the particles, which can be described by the Maxwell stress tensor as follows [29]:

$$
\boldsymbol{\sigma}^{M}=\mu_{0}\left[\mathbf{H} \mathbf{H}^{\mathrm{T}}-\frac{1}{2}|\mathbf{H}|^{2} \mathbf{I}\right]
$$

where I represents the second-order unit tensor, and $\mu_{0}$ represents the relative magnetic permeability of particles.

In the solid mechanical module, the ferromagnetic particles and rubber matrix are both assumed to be an elastic isotropic material whose stiffness and deformation depends on Young's modulus $(E)$ (nearly no magnetic effect), Poisson ratio $(v)$, and the specific parameter values listed in Table 1. Equation $\mathbf{U}=(u, v)$ is used to describe the displacement field of the MRE. The boundary condition is chosen in such a way that $u=0$ for boundary $l_{a d}$, and $v=0$ for boundary $l_{a b}$, whereas the remaining boundaries can be moved freely. Then, the magnetic force obtained in the magnetic module is applied to the ferromagnetic particle surface as the boundary loads and surface microstructure deformation of MRE can be obtained.

\subsection{Numerical results and discussion}

Using the magneto-mechanical coupling algorithm, a series of numerical simulations was carried out, the magnetic field $(\mathbf{H})$, particle volume fractions $(\phi)$, and initial rubber matrix modulus $\left(E_{0}\right)$ were selected to investigate their effects on the surface roughness change in MRE.

The simulation results of the MRE model under an applied magnetic field of $2.5 \mathrm{~T}$ are shown in Fig. 3 . Figure 3(a) gives the results of the magnetic flux density distribution in the simulation domain. Figure 3(b) shows the stress field of MRE induced by the magnetic field. These results illustrate that the magnetized particles in the matrix would interact with each other through the magnetic force and create a magnetic-

Table 1 Material properties.

\begin{tabular}{cccc}
\hline & \multicolumn{2}{c}{ Mechanical property } & Magnetic property \\
\cline { 2 - 4 } & $\begin{array}{c}\text { Young's } \\
\text { modulus }(E)\end{array}$ & $\begin{array}{l}\text { Poisson's } \\
\text { ratio }(v)\end{array}$ & $\begin{array}{c}\text { Relative } \\
\text { permeability }\left(\mu_{\mathrm{r}}\right)\end{array}$ \\
\hline Iron particles & $200 \mathrm{GPa}$ & 0.29 & 200 \\
Silicone rubber & $0.5 \mathrm{MPa}$ & 0.49 & 1 \\
\hline
\end{tabular}


(a)

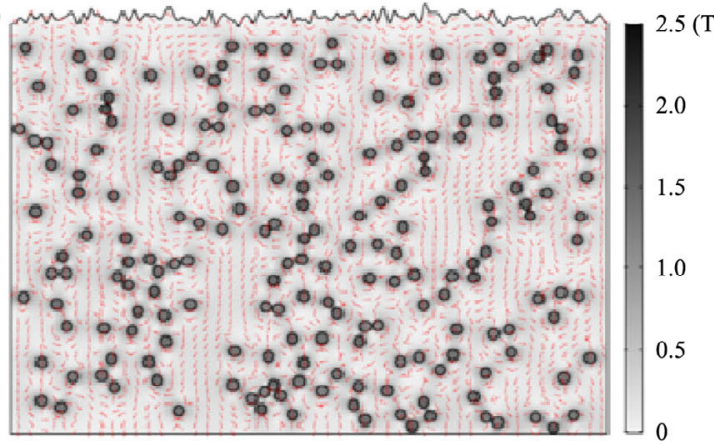

(b)

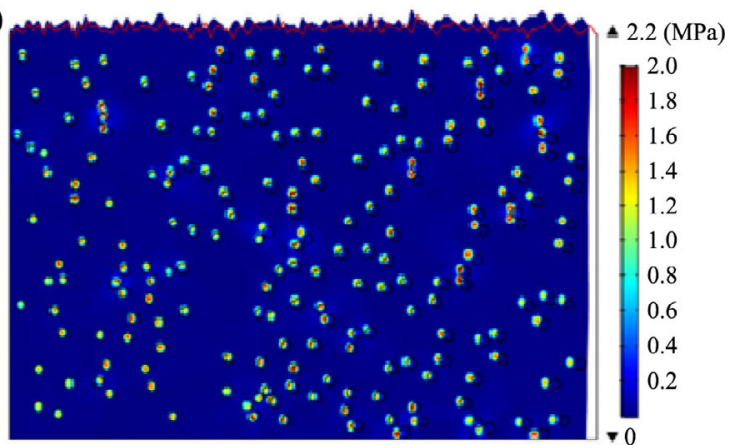

Fig. 3 Simulation results of the proposed model: (a) magnetic flux density distribution and (b) stress field.

induced stress in MRE, leading to a minor change in the distribution of particles in the matrix and causing the deformation of MRE. The deformations of MRE extend along the applied magnetic field and shrink in the direction perpendicular to the magnetic field, which is consistent with the experimental results of Ref. [30].

After the post-treatment of the simulation results, the surface profiles of MRE under the magnetic field is obtained. According to Eq. (2), the surface roughness of the deformed surface profile is calculated to be $2.41 \mu \mathrm{m}$, as shown in Fig. 4 .

This demonstrates that the surface of MRE has changed under a magnetic field, which is mainly due to the fact that after the application of the magnetic field, the ferromagnetic particles are immediately magnetized, creating attractive or repulsive forces between them, which results in varying degrees of surface variation. The relative heights of the peaks on the surface are diminished by the attraction of the magnetic field. In addition, some particles on the surface of MRE attract each other, filling up the valleys and resulting in the surface roughness change, which was shown in Fig. 5.

According to the above analysis, the interaction between ferromagnetic particles in MRE under the action of the magnetic field is the main cause of the surface changes. Furthermore, the effect of different particle volume fractions on the surface change will be explored. The relative roughness change $R a_{\mathrm{r}}$ is used to indicate the degree of surface changes, which

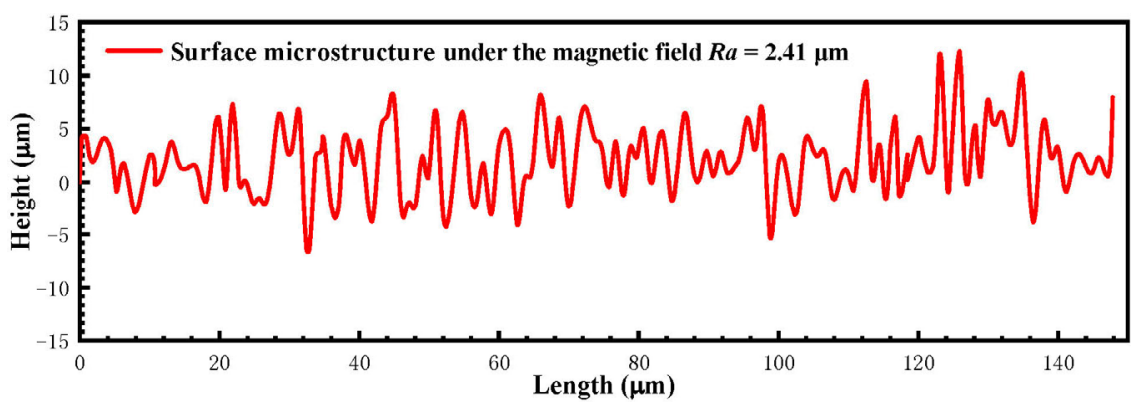

Fig. 4 The new surface micro-profile under the magnetic field is applied on.

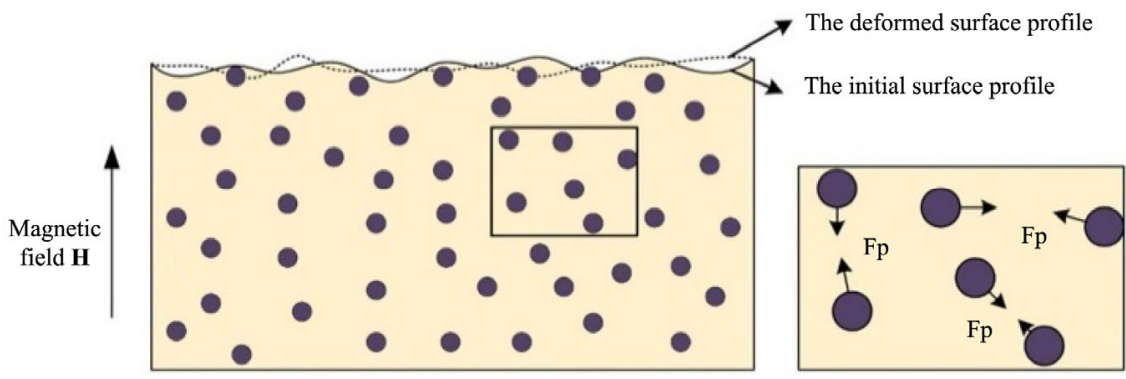

Fig. 5 Interaction of ferromagnetic particles with magnetic field. 
can be expressed as: $R a_{\mathrm{r}}=\left(R a_{\mathrm{m}}-R a\right) / R a$, where $R a_{\mathrm{m}}$ is the roughness under a magnetic field. Because the elasticity of MRE is weakened gradually with the sharp increase in the volume fraction of ferromagnetic particles, MRE has an upper limit on the ferromagnetic particle volume fraction, which is usually not greater than $20 \%$.

Figure 6 shows the simulation results of the relative roughness changes as a function of the particle volume fractions. It can be seen that the surface roughness of MRE with different particle volume fractions changes after the magnetic field is applied.

Based on the simulation results in Fig. 6, the surface change of MRE with particle volume fraction is divided into three stages. In stage I, the particle volume fraction is small, and the interaction between them is weak, which leads to a low degree of surface change. In stage II, with the increase in the particle volume fraction, the interaction between particles becomes stronger under the same magnetic field, and the surface change is more significant. It is worth noting that when the particle volume fraction is within $7.5 \%-9 \%, R a_{\mathrm{r}}$ can have a maximum absolute value. In stage III, the particle content continues to increase, but the degree of surface change is slightly decreased. It is considered that the increase in particle content can increase the interaction force between particles; however, it also increases the modulus of MRE and leads to a more difficult deformation. Therefore, it is inferred that the change in surface morphology with the particle volume fraction is a balance between the interaction

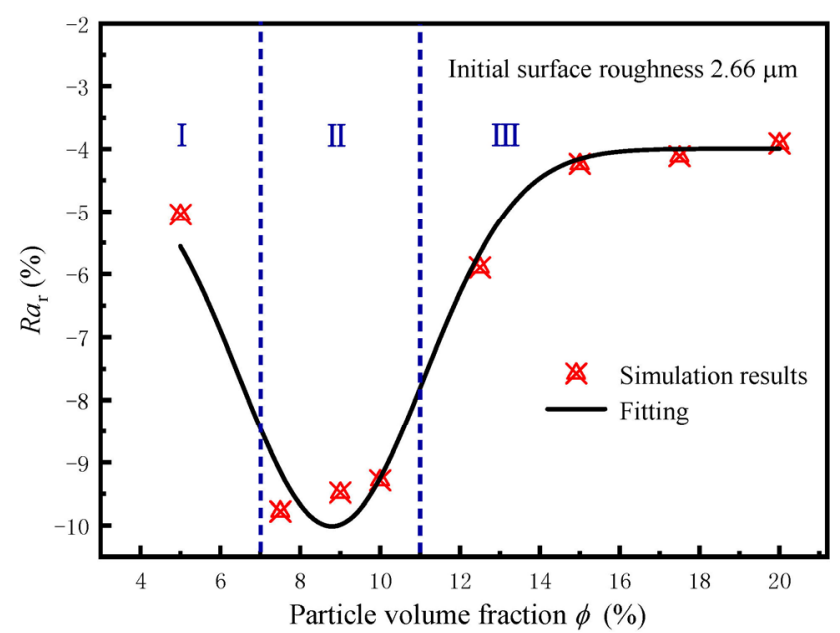

Fig. 6 Magnetic induced relative roughness $R a_{\mathrm{r}}$ with particle volume fraction. force and the modulus change caused by the increase in particles.

The MRE model with particle volume fraction of $9 \%$ is selected. By applying different magnetic fields, the influence of magnetic field intensity on the surface roughness of MRE is further explored. The simulation results are shown in Fig. 7.

The results show that the surface roughness of MRE changes significantly from an initial roughness of $2.42-2.66 \mu \mathrm{m}$ when the applied magnetic field strength reaches $1.25 \mathrm{~T}$ (Fig. 7). However, as the magnetic field further increases, the surface roughness of MRE will undergo little change. This phenomenon is related to the magnetization characteristics of magnetic particles. As the magnetization of the particles gradually reaches saturation, the increase in the magnetic field does not continue to produce significant changes.

The matrix modulus is an important factor that affects the mechanical properties of materials. The MRE prepared from different rubber materials has a different initial modulus, which may lead to different deformations on the surface under the same magnetic field. The relationship between the matrix modulus and the change in the surface microstructure under the same magnetic field is investigated by changing the matrix modulus. The simulation results are shown in Fig. 8, which illustrate that the relative variation in the surface roughness decreases with the increase in the modulus of the rubber matrix. The reason is that with the increase in the modulus of the rubber matrix,

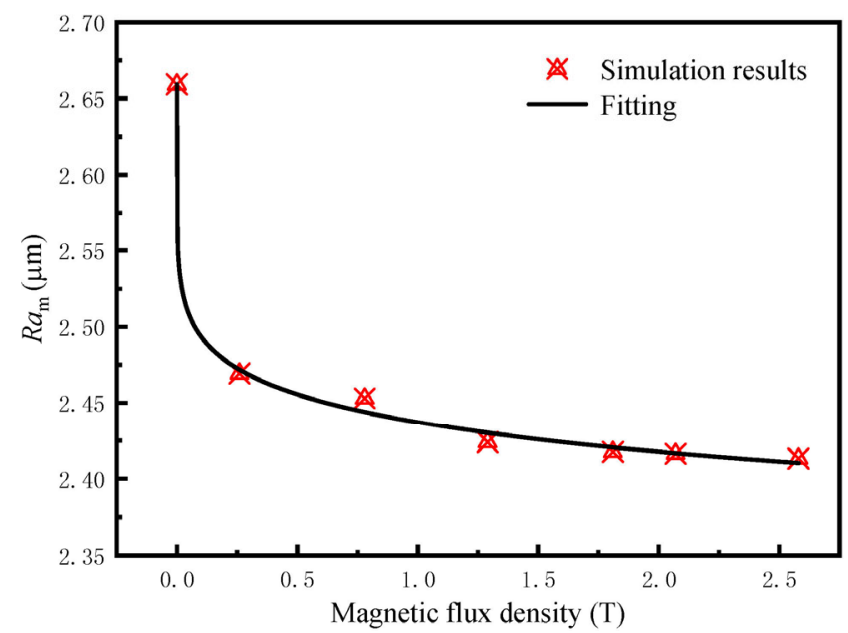

Fig. 7 Surface roughness of MREs change with magnetic field strength. 


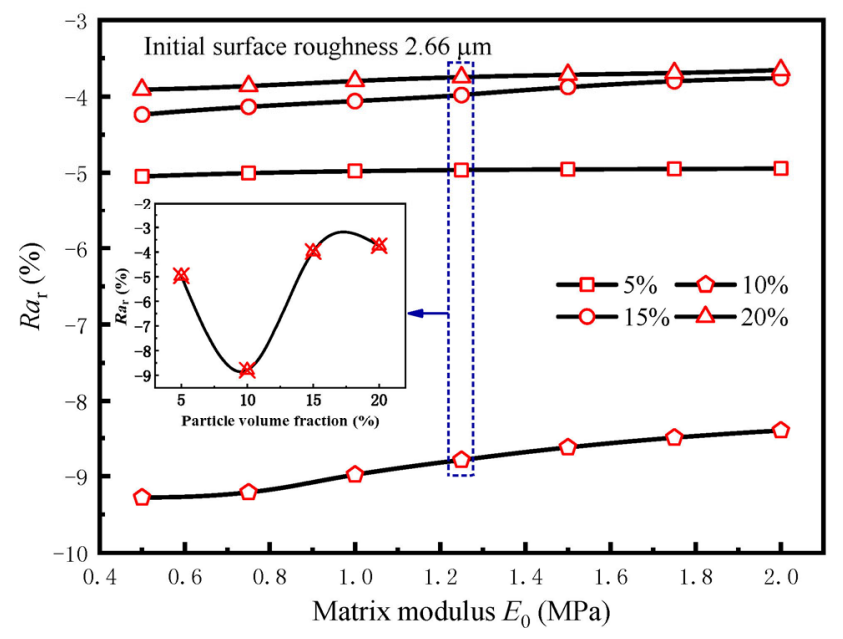

Fig. 8 Field-induced surface roughness of MREs as a function of matrix modulus for different particle volume fractions of $5 \%$, $10 \%, 15 \%$, and $20 \%$.

its ability to resist deformation increases, resulting in a decrease in the degree of surface deformation.

Through the FE, we analyzed the interaction between the ferromagnetic particles and between the particles and the rubber matrix from a microscopic point of view. We also explained the reason for the change in surface roughness. It is found that the particle volume fraction, the magnetic field strength, and the initial modulus of the matrix affect the change in the surface roughness.

\section{Materials and experiment study}

On the basis of numerical analysis, the surface topography of MRE under a magnetic field is further scanned by the white light interferometer. At the same time, a sliding friction experimental platform is built to explore how the surface changes affect the tribological characteristics of MRE.

\subsection{Preparation of the MRE samples}

The materials used to fabricate the isotropic MRE samples include the carbon iron particle (Beijing Xing Rong Yuan Technology Co., Ltd., China) whose diameter is $5 \mu \mathrm{m}$ and silicon rubber (HT-18; Shanghai Tongshuai Co., Ltd., China), which is made up of part $A$ and part $B$ with a mass ratio of 10:1. We also used polydimethylsiloxane (PDMS; $50 \mathrm{cp}$ two methyl silicone oil; Dow Corning, USA) as a diluting agent and $\mathrm{KH} 570$ as a silane coupling agent. Before the fabrication, we first diluted Part A of the silicone rubber using a diluting agent; then we pre-treated the carbon iron particles (referred to as CIPs) using KH570 to help the CIPs bond tightly with silicone rubber. After the pre-treatment, we mixed the CIPs and Part A, and dispersed them by ultrasonic radiation. Then, we mixed them with silicone rubber Part $B$, followed by degassing the mixture in a vacuum and transferring the mixture into a mold to form a typical sample. In this study, MREs with particle volume fractions of $5 \%, 10 \%, 15 \%$, and $20 \%$ are prepared. The materials used in the experiment are shown in Table 2, and the preparation process is shown in Fig. 9.

The internal structure of the prepared isotropic MRE sample is observed by scanning electron microscopy (SEM), which is illustrated in Fig. 10. It can be clearly seen that the ferromagnetic particles are spherical and randomly distributed in the rubber matrix.

\subsection{The surface observation experiment}

To observe the surface microstructure changes of the MRE under a magnetic field, a white light interference experiment system is developed as shown in Fig. 11. A white light interferometer (Counter GT-K) is applied to obtain the three-dimensional surface morphology of MRE samples, including surface topography, roughness, peaks, and valleys. A magnetic field generator, which can produce different magnetic field strengths at the observing interface, is installed right below the MRE when taking the image pictures. A PC is connected to the white light interferometer to store and process the image data.

In the experimental process, the samples are placed on the center of the magnetic field generator. They are first observed without the magnetic field, and then the surface is pictured under a magnetic field. Because of

Table 2 Compositions of MRE samples.

\begin{tabular}{ccccc}
\hline $\begin{array}{c}\text { Test } \\
\text { sample }\end{array}$ & $\begin{array}{c}\text { Particle } \\
\text { volume } \\
\text { fraction }\end{array}$ & $\begin{array}{c}\text { Part A of } \\
\text { the rubber } \\
\text { matrix }(\mathrm{g})\end{array}$ & $\begin{array}{c}\text { Part B of } \\
\text { the rubber } \\
\text { matrix }(\mathrm{g})\end{array}$ & CIPs (g) \\
\hline $\begin{array}{c}\text { Isotropic } \\
\text { MRE }\end{array}$ & $10 \%$ & 40 & 4 & 15.149 \\
& $15 \%$ & 40 & 4 & 31.981 \\
& $20 \%$ & 40 & 4 & 50.794 \\
\hline
\end{tabular}




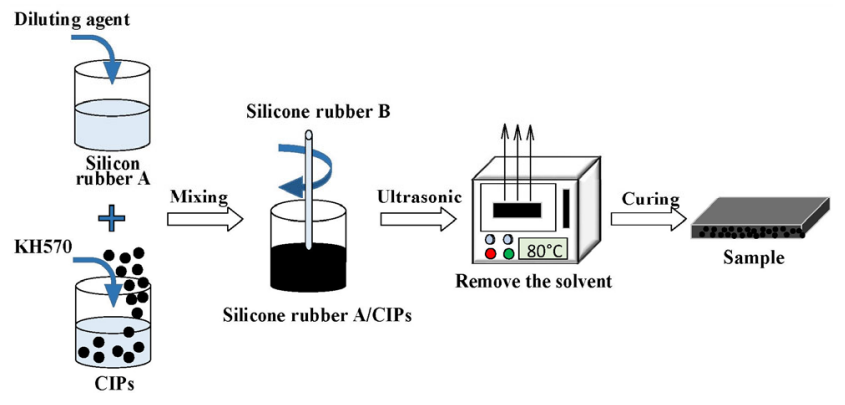

Fig. 9 The preparation process of MRE samples.
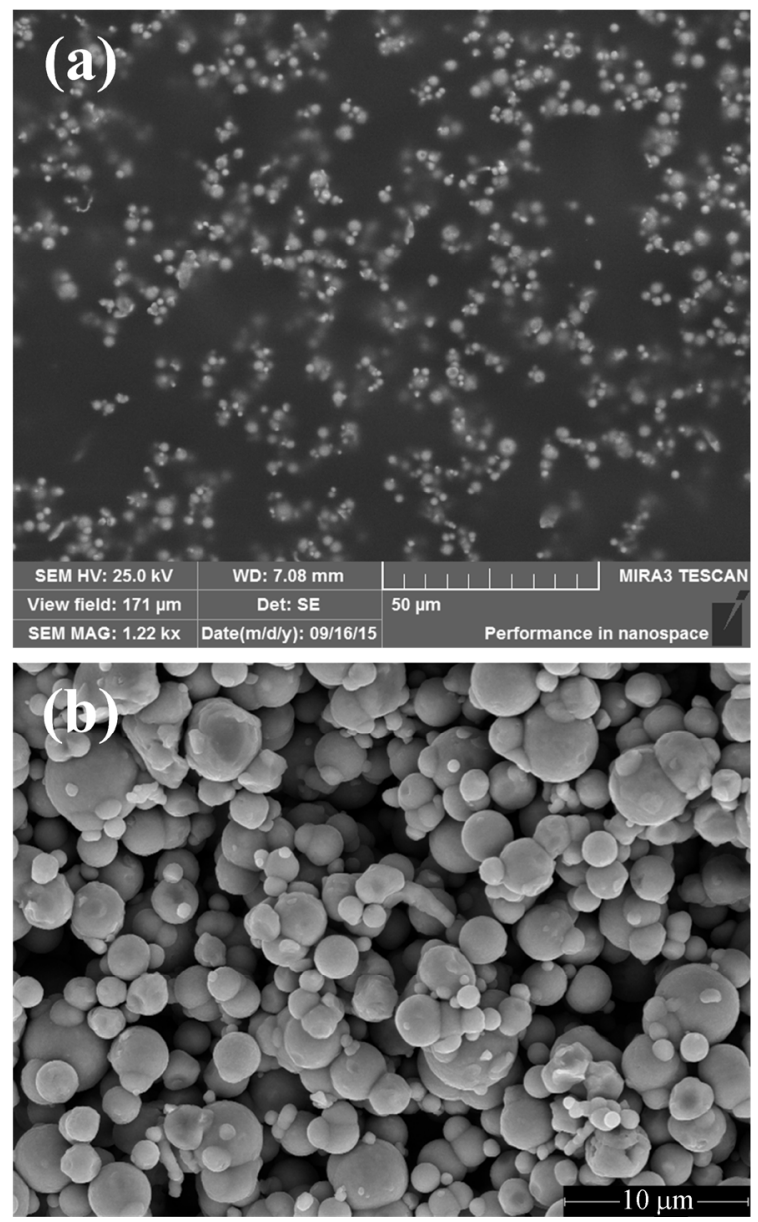

Fig. 10 (a) SEM micrograph of the MRE sample and (b) the microstructure of CIPs.

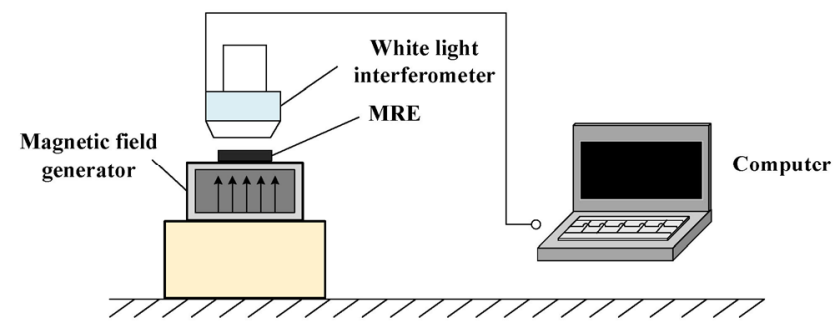

Fig. 11 Schematic of the white light interference experiment system. the high magnification of the white light interferometer, its field of view is limited. Therefore, during the observation process, multiple different positions on the surface of the MRE sample are selected for scanning, so as to avoid accidental results in the experiment. Figure 12 shows the morphology of MRE whose volume fraction is $10 \%$ with and without a magnetic field.

Figure 12(a) shows the initial surface of prepared MRE samples is not smooth. There are many red convex peaks and blue concave valleys distributed on the surface. After application of the magnetic field, the peaks were significantly reduced, as shown in Fig. 12(b). A two-dimensional profile of a threedimensional surface is selected for observation, as shown in Fig. 12(c), which shows that the surface morphology of MRE changes significantly under the influence of magnetic field. Furthermore, the scanning results of MREs with different particle volume fractions under a magnetic field strength of $500 \mathrm{mT}$ are analyzed and processed, and the average surface roughness $R a$ is obtained, as shown in Fig. 13.

As can be seen from Fig. 13, the surface roughness of all MREs decreases after applying magnetic field, which is consistent with the results of numerical analysis. When the volume particle volume fraction is $10 \%$, the surface roughness change of MRE is the largest, at approximately $14.6 \%$.

\subsection{The sliding friction experiment}

In the previous section, both theoretical analysis and microscopic experimental observations show that the surface microstructure of MRE changes regularly under the action of magnetic field. Furthermore, we verify the effect of surface changes on friction performance through macroscopic friction tests. The tribological properties of MRE samples with different particle volume fractions are tested under different magnetic field conditions, while maintaining external factors, such as temperature and relative humidity, unchanged. A sliding friction test platform is designed and built, as shown in Fig. 14 .

The experimental platform is mainly composed of a stepping motor, a linear guide, a pull pressure sensor, a magnetic field application device, and a base. During the experiment, a constant normal 



Fig. 12 White light interferometer test results of a typical MRE: (a) without magnetic field, (b) with magnetic field (500 mT), and (c) change of two-dimensional contour.

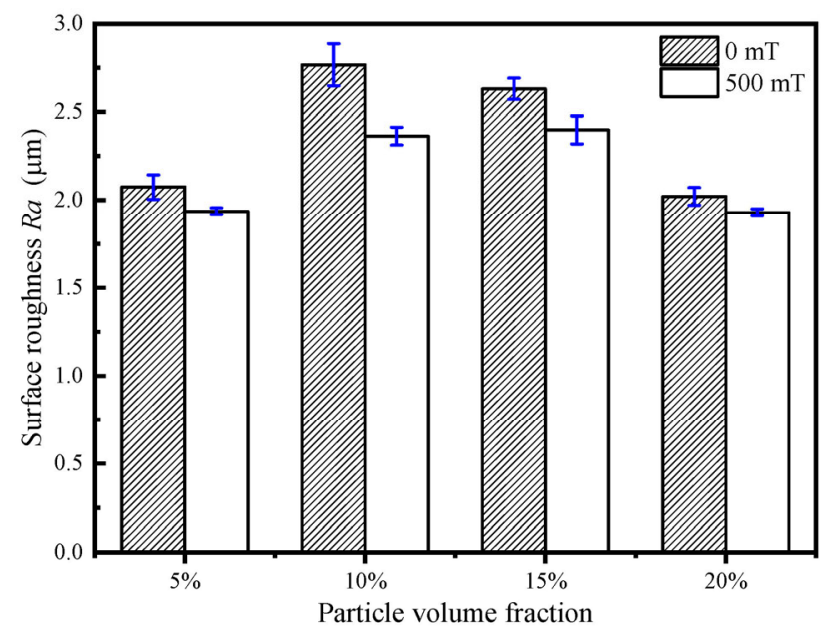

Fig. 13 The surface roughness of MRE with/without magnetic field.

pressure is provided by the copper block. The stepping motor ensures a uniform linear motion of the friction pairs during the friction test, with a uniform velocity of motion of $0.01 \mathrm{~mm} / \mathrm{s}$ and a movement time of $180 \mathrm{~s}$. The pressure sensor is used to measure the friction force with/without the magnetic field. The friction coefficient is obtained using the formula $f=F / N$, where $F$ is the friction force and $N$ is the normal pressure. Each experiment is repeated three times, and the results are averaged over three experiments.
We have carried out experimental tests on a variety of MRE samples. Figure 15 shows the variation of the friction performance of the MRE samples under a different magnetic field, with the particle volume fractions of $5 \%, 10 \%, 15 \%$, and $20 \%$ of which the normal force is $1.97 \mathrm{~N}$.

As can be seen from Fig. 15, the curve of friction force changing with time during the testing process. The results show that the friction force on the MRE surface decreases significantly after the magnetic field is applied, and the decreasing trend becomes more and more significant with the increase in the magnetic field, which indicates that the magnetic field has an effect on the friction properties of MRE. Moreover, the average friction coefficients of each component MRE under different magnetic field conditions are obtained through Coulomb theory, and the statistical results are shown in Fig. 16.

Figure 16 shows that when the other external factors remain unchanged, the MRE friction coefficient decreases with the variation in the magnetic field under the same pressure, which is consistent with the change trend of surface roughness under the effect of the magnetic field. When the particle volume fraction is $10 \%$, the magnetic field intensity is $400 \mathrm{mT}$, and the range of the adjustable friction coefficient of MRE is 

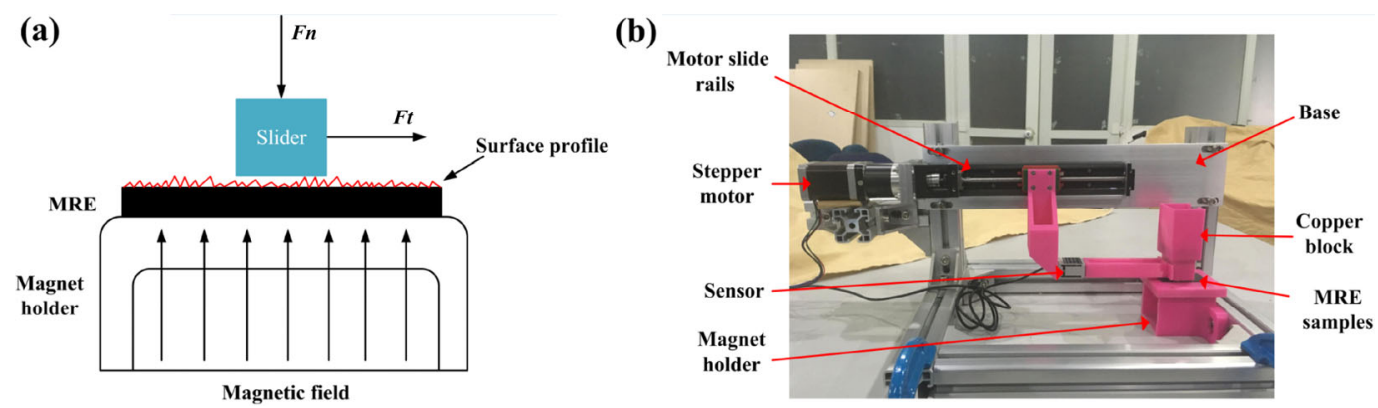

Fig. 14 The schematic and the real photo of the friction test platform: (a) the schematic and (b) the real photo.
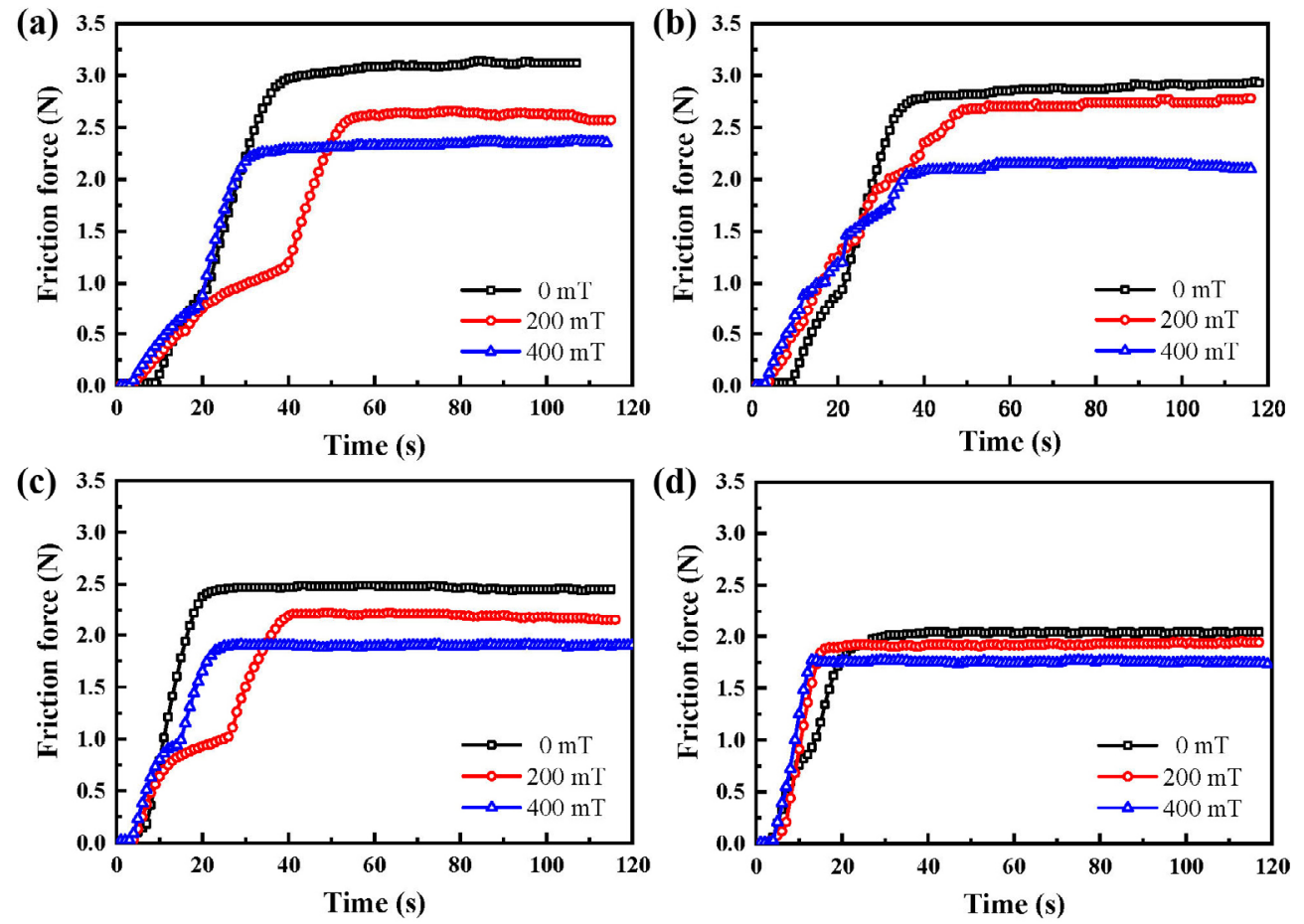

Fig. 15 The curves of friction with time: (a) $5 \%$, (b) $10 \%$, (c) $15 \%$, and (d) $20 \%$.

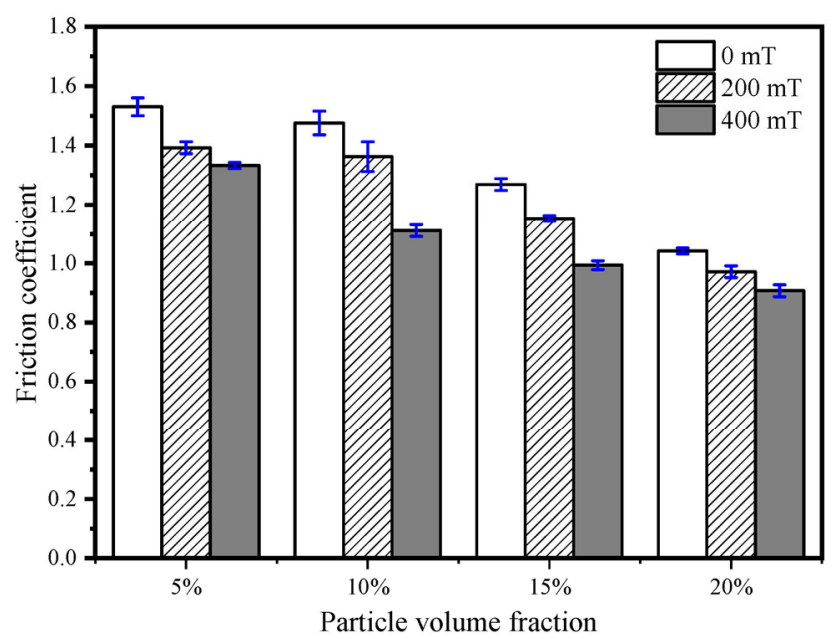

Fig. 16 Frictional coefficient of MRE with different volume fractions under different magnetic fields. the largest, which is $24.7 \%$. This coincides with the theoretical simulation analysis of Section 2 that when the volume fraction is within $7.5 \%-9 \%$, the relative change rate is the most significant. At the same time, the white light interference experiment from Section 3 also shows that the surface roughness of MRE decreases most when the particle volume fraction is about $10 \%$. Therefore, it is evident that the change in the surface roughness is an important factor that leads to the change in the friction properties of the MRE friction properties. By adjusting the change in surface roughness under the action of a magnetic field, the friction properties of MRE can be effectively regulated.

In addition to the surface morphology of MRE under a magnetic field, its mechanical properties will 
also change. Whether the magnetic-controlled friction characteristics of MRE are affected by the coupling of multiple factors will be further explored.

\section{Conclusions}

(1) Based on the magnetization characteristics of ferromagnetic particles in MRE, a micro-finite element model of MRE was established to analyze the surface morphology changes in MRE under a magnetic field. The initial surface microstructures of MRE were taken into account and characterized by the $\mathrm{W}-\mathrm{M}$ fractal function. The numerical results show that the magnetic field can control the surface roughness of MRE. However, the degree of change is affected by the particle volume fraction, rubber matrix modulus, and magnetic field strength. With the increase in magnetic field intensity and decrease in matrix modulus, the surface roughness of MRE decreases continuously. Meanwhile, the volume fraction with the best magneticinduced relative roughness change is within $7.5 \%-9 \%$.

(2) On the basis of numerical analysis, the MRE samples with different particle volume fractions were prepared, and the surface morphology changes of MRE under a magnetic field were observed by white light interferometry. At the same time, a sliding friction experimental platform was set up to measure the sliding friction characteristics of MRE samples under different magnetic fields, so as to confirm that the change in the surface morphology will affect the friction characteristics of MRE samples. The results of the white light interference experiment show that the height of the convex peaks on the MRE surface decreases due to the effect of the magnetic field; thus, the surface roughness decreases. The maximum reduction is approximately $14.6 \%$ with a particle volume fraction of $10 \%$. This is consistent with the simulation results, which verifies the validity of the FE model. The results of friction experiments show that because of the decrease in the MRE surface roughness, the friction coefficients decrease with the application and increase in the magnetic field. With a magnetic field of $400 \mathrm{mT}$, the friction coefficient of MRE with a particle volume fraction of $10 \%$ can decrease by $24.7 \%$. In this study, it is verified that the change in the surface roughness with the action of the magnetic field is an important factor, leading to the change in friction performance, which provides a new method for the friction control of MRE.

\section{Acknowledgements}

This work was supported by the National Natural Science Foundation of China (No. 11572320), Science and Technology Research Project of Chongqing Municipal Education Commission (No. KJQN201800644), and Special Key Project of Technological Innovation and Application Development in Chongqing (cstc2019jscxfxyd0005). The authors thank professor Xiaojie WANG from Institute of Advanced Manufacturing Technology, Hefei Institutes of Physical Science, Chinese Academy of Sciences and associate professor Shiwei CHEN from Chongqing Institute of Science and Technology for the support and fruitful discussions.

Open Access This article is licensed under a Creative Commons Attribution 4.0 International License, which permits use, sharing, adaptation, distribution and reproduction in any medium or format, as long as you give appropriate credit to the original author(s) and the source, provide a link to the Creative Commons licence, and indicate if changes were made.

The images or other third party material in this article are included in the article's Creative Commons licence, unless indicated otherwise in a credit line to the material. If material is not included in the article's Creative Commons licence and your intended use is not permitted by statutory regulation or exceeds the permitted use, you will need to obtain permission directly from the copyright holder.

To view a copy of this licence, visit http:// creativecommons.org/licenses/by/4.0/.

\section{References}

[1] Jolly M R, Carlson J D, Muñoz B C, Bullions T A. The magnetoviscoelastic response of elastomer composites consisting of ferrous particles embedded in a polymer matrix. J Intell Mater Syst Struct, 7(6): 613-622 (1996)

[2] Chirila P E, Chirica I, Beznea E F. Damping properties of magnetorheological elastomers. Adv Mater Res, 1143: 247-252 (2017)

[3] Chen S W, Wang X J, Zhang Z, Mu W J, Li R. Optimal design of laminated-MRE bearings with multi-scale model. Smart Mater Struct, 25(10): 105037 (2016) 
[4] Li R, Mu W J, Zhang L Y, Wang X J. Design and testing performance of a magneto-rheological elastomer isolator for a scaled bridge system. J Intell Mater Syst Struct, 29(2): 171-182 (2017)

[5] Lindler J E, Dimock G A, Wereley N M. Design of a magnetorheological automotive shock absorber. In Proceedings Volume 3985, Smart Structures and Materials 2000: Smart Structures and Integrated Systems, Newport Beach, United States, 2000: 426-437.

[6] Ginder J M, Schlotter W F, Nichols M E. Magnetorheological elastomers in tunable vibration absorbers. In Proceedings Volume 4331, Smart Structures and Materials 2001: Damping and Isolation, Newport Beach, United States, 2001: 103-110.

[7] Geng J L, Wang C P, Zhu H L, Wang X J. Effect of the carbonyl iron particles on acoustic absorption properties of magnetic polyurethane foam. In Proceedings Volume 10596, Behavior and Mechanics of Multifunctional Materials and Composites XII, Denver, United States, 2018: 116-126.

[8] Li R, Zhou M J, Wang M L, Yang P A. Study on a new self-sensing magnetorheological elastomer bearing. $A I P A d v$, 8(6): 065001 (2018)

[9] Yu M, Yang P A, Fu J, Liu S Z, Qi S. Study on the characteristics of magneto-sensitive electromagnetic waveabsorbing properties of magnetorheological elastomers. Smart Mater Struct, 25(8): 085046 (2016)

[10] Sedlacik M, Mrlik M, Babayan V, Pavlinek V. Magnetorheological elastomers with efficient electromagnetic shielding. Compos Struct, 135: 199-204 (2016)

[11] Lee D W, Lee K, Lee C H, Kim C H, Cho W O. A study on the tribological characteristics of a magneto-rheological elastomer. J Tribol, 135(1): 014501 (2012)

[12] Shaha K P, Pei Y T, Martinez-Martinez D, De Hosson J T $\mathrm{M}$. Influence of hardness and roughness on the tribological performance of TiC/a-C nanocomposite coatings. Surf Coat Technol, 205(7): 2624-2632 (2010)

[13] Zhang Y, Dong M, Gueye B, Ni Z H, Wang Y J, Chen Y F. Temperature effects on the friction characteristics of graphene. Appl Phys Lett, 107(1): 011601 (2015)

[14] Yoo S S, Kim D E. Effects of vibration frequency and amplitude on friction reduction and wear characteristics of silicon. Tribol Int, 94: 198-206 (2016)

[15] Feng D, Shen M X, Peng X D, Meng X K. Surface roughness effect on the friction and wear behaviour of acrylonitrilebutadiene rubber (NBR) under oil lubrication. Tribol Lett, 65(1): 10 (2017)

[16] Lian C L, Lee K H, Lee C H. Friction and wear characteristics of magneto-rheological elastomers based on silicone/ polyurethane hybrid. $J$ Tribol, 137(3): 031607 (2015)

[17] Lian C L, Lee K H, Lee C H. Friction and wear characteristics of magnetorheological elastomer under vibration conditions. Tribol Int, 98: 292-298 (2016)

[18] Lian C L, Lee K H, Lee C H. Effect of temperature and relative humidity on friction and wear properties of silicone-based magnetorheological elastomer. Tribol Trans, 61(2): 238-246 (2018)

[19] Gong X L, Liao G J, Xuan S H. Full-field deformation of magnetorheological elastomer under uniform magnetic field. Appl Phys Lett, 100(21): 211909 (2012)

[20] Lee S, Yim C, Kim W, Jeon S. Magnetorheological elastomer films with tunable wetting and adhesion properties. ACS Appl Mater Interfaces, 7(35): 19853-19856 (2015)

[21] Sorokin V V, Sokolov B O, Stepanov G V, Kramarenko E Y. Controllable hydrophobicity of magnetoactive elastomer coatings. J Magn Magn Mater, 459: 268-271 (2018)

[22] Sánchez P A, Minina E S, Kantorovich S S, Kramarenko E $Y$. Surface relief of magnetoactive elastomeric films in a homogeneous magnetic field: Molecular dynamics simulations. Soft Matter, 15(2): 175-189 (2019)

[23] Chen S W, Li R, Li X, Wang X J. Magnetic field induced surface micro-deformation of magnetorheological elastomers for roughness control. Front Mater, 5: 76 (2018)

[24] Danas K, Kankanala S V, Triantafyllidis N. Experiments and modeling of iron-particle-filled magnetorheological elastomers. J Mech Phys Solids, 60(1): 120-138 (2011)

[25] Martin J E, Anderson R A, Read D, Gulley G. Magnetostriction of field-structured magnetoelastomers. Phys Rev E Stat Nonlin Soft Matter Phys, 74(5): 051507 (2006)

[26] Yin X, Komvopoulos K. An adhesive wear model of fractal surfaces in normal contact. Int $J$ Solids Struct, 47(7-8): 912-921 (2010)

[27] Zhang X H, Xu Y, Jackson R L. An analysis of generated fractal and measured rough surfaces in regards to their multi-scale structure and fractal dimension. Tribol Int, 105: 94-101 (2017)

[28] Chen W W, Sun L Y, Li X H, Wang D F. Numerical investigation on the magnetostrictive effect of magnetosensitive elastomers based on a magneto-structural coupling algorithm. Smart Mater Struct, 22(10): 105012 (2013)

[29] Ly H V, Reitich F, Jolly M R, Banks H T, ItoK. Simulations of particle dynamics in magnetorheological fluids. J Comput Phys, 155(1): 160-177 (1999)

[30] Yi H, Hong W, Faidley L E. Field-stiffening effect of magneto-rheological elastomers. Int J Solids Struct, 50(14-15): 2281-2288 (2013) 


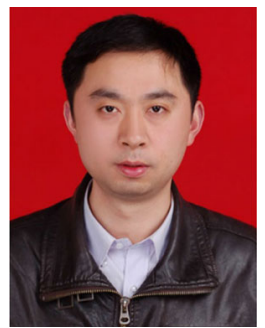

Rui Li. He received his B.S. degree in 1999 from Chongqing University of Technology, China, and received his M.S. degree and Ph.D. degree from Chongqing University, China,

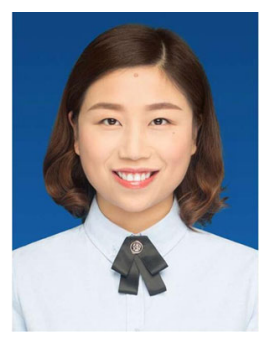

Xi LI. She received her B.S. degree in electrical engineering and automation form Chongqing University of Posts and Telecommunications, China, in 2016. She is studying for

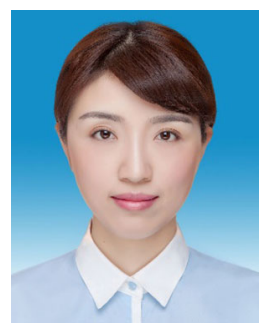

Yuanyuan Li. She is currently a senior engineer of Chongqing University of Posts and Telecommunications. She was invited to University of Alberta in Canada as

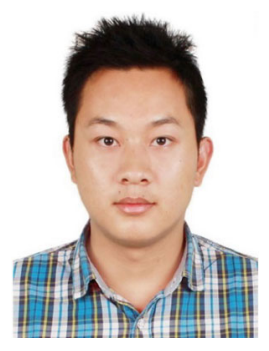

Ping-an YANG. He received his B.S. degree in electrical engineering and automation from Chongqing University of Posts and Telecommunications, China, in 2012, and his Ph.D. degree in instrument science and technology from Chongqing



Jiushan LIU. He received his B.S. degree in electrical engineering and automation from Chongqing University of Posts and Telecommunications, China, in 2015. He is in 2004 and 2009, respectively. His current position is a professor in Chongqing University of Posts and Telecommunications. His main research interests include intelligent detection technology, friction control, and intelligent mechanical structure system.

the M.S. degree at Chongqing University of posts and telecommunications, China. Her research interests are surface and friction characteristics of magnetorheological elastomer.

a visiting scholar. She is studying for the Ph.D. degree at China University of Mining and Technology, China. Her main research interests include information processing, intelligent vehicle, and image recognition.

University, China, in 2017. His current position is a lecturer in Chongqing University of Posts and Telecommunications. His main research interests include intelligent biomimetic composite materials, flexible sensor, electromagnetic shielding material, and structural design.

studying for the M.S. degree at Chongqing University of posts and telecommunications, China. His research interests are magnetron friction and adhesion properties of magnetorheological elastomer. 